DIFFERENTIAL GBOMETRY

BANACH CENTER PUBLICATIONS, VOLUME 12

PWN-POLISH SCIENTIFIC PUBLISHERS

WARSAW 1984

\title{
CAUCHY'S PROBLEM FOR BACH'S EQUATIONS OF GENERAL RELATIVITY
}

\author{
R. SCHIMMING
}

Department of Mathematics, Ernst Moritz Arndt University, Greifswald, G.D.R.

\section{Motivation}

From the variational point of view the most natural modifications of Einstein's equations are to be derived from Lagrangians

$$
L=k R+a_{0} R^{2}+a_{1}|\mathrm{Ric}|^{2}+a_{2}|\mathrm{Riem}|^{2}
$$

for a space-time metric

$$
g=g_{\alpha \beta} d x^{\alpha} d x^{\beta} \quad(\alpha, \beta=0,1,2,3) .
$$

We write

curvature tensor $=$ Riem $=R_{\alpha \beta \mu \nu} d x^{\alpha} \wedge d x^{\beta} d x^{\mu} \wedge d x^{\nu}$,

Ricci tensor $=$ Ric $=R_{\alpha \beta} d x^{\alpha} d x^{\beta}$,

scalar curvature $=R$,

conformal curvature tensor $=$ Weyl $=C_{\alpha \beta \mu \nu} d x^{\alpha} \wedge d x^{\beta} d x^{\mu} \wedge d x^{\nu}$,

$\nabla=d x^{\alpha} \nabla_{\alpha}=$ Levi-Civita covariant derivative.

Theories based on (1) have been discussed in the early times of general relativity and have recently gained renewed interest in the context of quantum gravity, elementary length, gauge field ideas, supergravity, singularity problems, nonconventional matter field equations (viz. fourth order equations or equations containing curvature terms). Note that the quantized version of a mixed theory (1) is renormalizable [9]. Einstein's theory on the one hand and purely quadratic theories on the other hand may be looked upon as limiting cases of (1).

The special choice due to R. Bach [1]

$$
L=\mid \text { Weyl }\left.\right|^{2}=\frac{1}{3} R^{2}-2 \mid \text { Ric }\left.\right|^{2}+\mid \text { Riem }\left.\right|^{2}
$$

implies the vacuum field equations

$$
B_{\alpha \beta} \equiv \nabla^{\mu} \nabla^{\nu} C_{\mu \alpha \beta \nu}-\frac{1}{2} C_{\mu \alpha \beta \nu} R^{\mu \nu}=0 .
$$

Bach's tensor $B_{\alpha \beta} d x^{\alpha} d x^{\beta}$ is tracefree and conformally invariant of weight -1 . It appears as an object of conformal geometry and in investigations on Huygens' 
principle for linear hyperbolic differential equations. Some classes of exact solutions of Bach's equations have been constructed [3], [4]. P. Günther studied the shock waves to (4) and qualitatively analysed Cauchy's problem [7], [6]. Our work is a continuation and refinement of [6].

\section{The constraints}

Let $(M, g)$ be a space-time with local coordinates $x^{\alpha}(\alpha=0,1,2,3)$ and $S \subset M$ a spacelike hypersurface with intrinsic coordinates $t^{i}(i=1,2,3)$, unit normal $n=n^{\alpha} \partial_{\alpha}$, and connecting quantities $x_{i}^{\alpha}=\partial x^{\alpha} / \partial t^{i}$. In Einstein's theory the first and second fundamental forms of $S$

$$
\begin{aligned}
\mathrm{I} & =g_{i j} d t^{i} d t^{j}=g_{\alpha \beta} x_{i}^{\alpha} d t^{i} x_{j}^{\beta} d t^{j}, \\
\mathrm{II} & =P_{t j} d t^{i} d t^{j}=\left(\nabla_{\alpha} n_{\beta}\right) x_{i}^{\alpha} d t^{i} x_{j}^{\beta} d t^{i}
\end{aligned}
$$

serve as Cauchy data. In Bach's theory we complete them by

$$
\begin{aligned}
& \text { III }=Q_{t j} d t^{i} d t^{j}=n^{\mu} x_{i}^{\alpha} x_{j}^{j} n^{\nu} C_{\mu \alpha \beta \nu} d t^{i} d t^{j}, \\
& \text { IV }=S_{\iota j} d t^{i} d t^{j}=n^{\mu} x_{i}^{\alpha} x_{j}^{\beta} \nabla^{\nu} C_{\mu \alpha \beta \nu} d t^{i} d t^{j} .
\end{aligned}
$$

Obviously, projection of an $M$-tensor of degree $p$ on the tetrad $\left(n, \partial / \partial t^{l}\right)$ decomposes it into $S$-tensors of degrees $0,1, \ldots, p$. We use a shorthand notation:

$$
\begin{gathered}
B_{n n}=n^{\alpha} n^{\beta} B_{\alpha \beta}, \quad B_{n 1}=n^{\alpha} x_{i}^{\beta} B_{\alpha \beta}, \quad B_{i j}=x_{i}^{\alpha} x_{j}^{\beta} B_{\alpha \beta}, \\
R_{i j k l}=x_{i}^{\alpha} x_{j}^{\beta} x_{k}^{\mu} x_{l}^{\nu} R_{\alpha \beta \mu \nu}, \\
R_{i j k n}=x_{i}^{\alpha} x_{j}^{\beta} x_{k}^{\mu} n^{\nu} R_{\alpha \beta \mu \nu,}
\end{gathered}
$$

etc. Gauss-Codazzi equations tell us that $R_{i j k l}, R_{i j k n}, C_{i j k l}, C_{i j k n}$ are $S$-intrinsic differential expressions in I, II. This can be established by means of Van der WaerdenBortolotti's $D_{i}$-symbols: The $D_{i}$ act as $x_{i}^{\alpha} \nabla_{\alpha}$ on $M$-tensors and as $I$-covariant derivatives on $S$-tensors. Note that we raise Latin indices $i, j, k, \ldots$ by means of $\left(g^{\prime j}\right):=\left(g_{i j}\right)^{-1}$.

THEOREM 1. The tetrad components $B_{n n}, B_{n i}$ taken on $S$ are $S$-intrinsic differential expressions in I, II, III, IV, viz.

$$
\begin{aligned}
& B_{n n}=D^{l}\left(D^{j} Q_{i j}+P^{j k} C_{l j k n}\right)-Q^{i j}\left(Q_{i j}+R_{i k j}^{k}\right)+P^{i j} S_{i j} \\
& B_{n i}=-D^{j} S_{i j}+\frac{1}{2} D^{j}\left(D^{k} C_{i j k n}+3 P_{l}^{k} Q_{j k}-\right.\left.P_{j}^{k} Q_{i k}\right)+ \\
&+P_{l}^{j} P^{k l} C_{l j k n}-Q^{j k} D_{l} P_{j k}-C_{i j k n} R_{l}^{j ! k} .
\end{aligned}
$$

We developped three methods for reducing the number of unknown functions and transforming the equation system $B_{n n}=0, B_{n i}=0$ into an elliptic one.

1. Method. Application of the closed range theorem in Sobolev-Hilbert spaces $W^{l}=W_{2}^{l}=W_{2}^{l}(S)$. Let $(S, I)$ be closed and $C^{\infty}, l \geqslant 2, m \geqslant 1$. Then for each $Q_{i j} \in W^{l}$ there exist $\hat{Q_{i j}} \in W^{l}, u \in W^{l+2}$ such that

$$
Q_{t \jmath}=\hat{Q}_{i j}+D_{i} D_{\jmath} u-\frac{1}{3} g_{i j} \Delta u, \quad D^{l} D^{J} \hat{Q}_{t \jmath}=0
$$


Also for each $S_{i j} \in W^{m}$ there exist $\hat{S}_{i j} \in W^{m}, v_{i} \in W^{m+1}$ such that

$$
S_{i j}=\hat{S}_{i j}+D_{(i)} v_{j)}-\frac{1}{3} g_{i j} D^{k} v_{k}, \quad D^{j} \hat{S}_{i j}=0 \text {. }
$$

The splittings (8), (9) make (7) Petrovskij-elliptic in $\left(u, v_{i}\right)$ with orders $(4,2)$ :

$$
3 B_{n n}=2 \Delta^{2} u+\ldots, \quad 6 B_{n i}=3 \Delta v_{i}+D_{i} D^{k} v_{k}+\ldots
$$

This procedure works with modifications also in other topological situations.

2. Method. Lapse and shift formalism. A family of spacelike hypersurfaces containing $S$ and additionally a timelike vector field

$$
\frac{\partial}{\partial t}=N \cdot n+X^{i} \frac{\partial}{\partial t^{i}}=N \cdot n+X
$$

defining lapse $N$ and shift $X$, are presupposed. Abbreviating time derivatives $\frac{\partial}{\partial t}$ by dots we obtain

$$
\begin{aligned}
2 N P_{l j} & =\dot{g}_{i j}-D_{(i} X_{j}, \\
2 N Q_{i j} & =D_{i} D_{j} N-\frac{1}{3} g_{i j} \Delta N+\ldots, \\
-2 N^{3} S_{i j} & =\text { tracefree part }\left(N X^{k} D_{k} D_{i} D_{\jmath} N+D_{(i} \ddot{X}_{\jmath}\right)+\ldots
\end{aligned}
$$

and as a conclusion the Petrovskij-elliptic system

$$
\begin{aligned}
3 N B_{n n} & =\Delta^{2} N+\ldots \\
9 N^{3} B_{n i} & =4 N X^{k} D_{k} D_{l} \Delta N+3 \Delta \ddot{X}_{i}+D_{l} D^{k} \ddot{X}_{k}+\ldots
\end{aligned}
$$

Only terms contributing to the Petrovskij-principal part are written down.

3. Method. Reduction of the dimension by means of symmetry assumptions. For $P_{i j}=0$ our system simplifies to

$$
\begin{aligned}
& B_{n n}=D^{i} D^{j} Q_{i j}-Q^{i j} Q_{i j}=0, \\
& B_{n i}=-D^{j} S_{i j}=0 .
\end{aligned}
$$

If additionally

$$
\begin{gathered}
g_{i j}=\delta_{l j}, \quad Q_{13}=0, \quad \partial_{3} Q_{i j}=0, \quad S_{13}=0, \quad \partial_{3} S_{i j}=0, \\
z:=t^{1}+i t^{2}, \quad 4 q:=Q_{12}+i Q_{11}, \quad 4 s:=S_{12}+i S_{11},
\end{gathered}
$$

then the constraints reduce to

$$
\Re \mathfrak{n}\left(\partial^{2} q / \partial \bar{z}^{2}-q^{2}\right)=0, \quad \partial s / \partial \bar{z}=0 .
$$

Special solutions of (16) can be found. The simplification (15) is only an examplea lot of other special solutions could be constructed.

\section{The evolution problem}

As is well known, the Ricci tensor splits into a hyperbolic part $\vee$ and into a gauge part $\wedge$ :

15* 


$$
\begin{aligned}
& \boldsymbol{R}_{\alpha \beta}=\check{R}_{\alpha \beta}+\hat{R}_{\alpha \beta}, \\
& \check{R}_{\alpha \beta}=\frac{1}{2} g^{\mu \nu} \partial_{\mu} \partial_{\nu} g_{\alpha \beta}+r_{\alpha \beta}\left(j^{1} g_{\mu \nu}\right), \\
& \hat{R}_{\alpha \beta}=-\nabla_{(\alpha} \Gamma_{\beta)} \quad \text { with } \quad \Gamma_{\alpha}:=g^{\prime \prime \nu} \Gamma_{\mu \nu \alpha} .
\end{aligned}
$$

The analogous splitting for

$$
2 B_{\alpha \beta}=\square\left(R_{\alpha \beta}-\frac{1}{0} R g_{\alpha \beta}\right)-\frac{1}{3} \nabla_{\alpha} \nabla_{\beta} R-\left(C_{\mu \alpha \beta \nu}+R_{\mu \alpha \beta \nu}-R_{\mu \alpha \alpha} g_{\beta \nu}\right) R^{\mu \nu}
$$

reads

$$
\begin{aligned}
& 2 \check{B}_{\alpha \beta}=\square \check{R}_{\alpha \beta}-\left(C_{\mu \alpha \beta \nu}+R_{\mu \alpha \beta \nu}-R_{\mu \alpha \alpha} g_{\beta \nu}\right) R^{\mu \nu}, \\
& 2 \hat{B}_{\alpha \beta}=\square \hat{R}_{\alpha \beta}-\frac{1}{6} g_{\alpha \beta} \square R-\frac{1}{3} \nabla_{\alpha} \nabla_{\beta} R .
\end{aligned}
$$

Here we treat the contractions $\Gamma_{\alpha}, \Gamma^{\alpha}$ of Christoffel symbols $\Gamma_{\mu v \alpha}, \Gamma_{\mu \nu}^{\alpha}$ as well as $\check{R}_{\alpha \beta}$, $\hat{R}_{\alpha \beta}$ formally like tensors and the jet symbol $j^{k}$ denotes the collection of all partial derivatives of orders $0,1, \ldots, k$. While $\hat{R}_{\alpha \beta}$ can be gauged away by the choice of harmonic coordinates

$$
\Gamma^{\alpha} \equiv-\square x^{\alpha}=0,
$$

in Bach's theory the gauge conditions read

$$
\text { harmonic gauge } \Gamma^{\alpha}=0, \quad \text { conformal gauge } R=0 \text {. }
$$

The informations contained in Cauchy's data on $S$ exhibit some algebraic structure:

$$
\begin{aligned}
& \text { minimal data } \oplus \text { gauge data }=\text { maximal data, viz. } \\
& (\text { I , II, III, IV }) \oplus(N, X) \oplus\left(j^{2} \Gamma^{\alpha}, j^{1} R\right)=\left(j^{3} g_{\alpha \beta}\right) .
\end{aligned}
$$

In the following a manifold is understood as Hausdorff, connected and paracompact. A space-time is a 4-dimensional manifold equipped with a lorentzian metric and a time orientation. We use local Sobolev-Hilbert spaces $W^{l}=W_{2}^{l}$.

Definition 1 . Let $(M, g)$ be a space-time and $S \subset M$ a spacelike hypersurface. A nonempty open set $U \subseteq M$ is called a Cauchy neighborhood over $S \cap U$ if every past (future)-directed causal curve $\gamma$ issuing from a point $x \in U \backslash S$ and without past (future) endpoint in $U$ intersects $S$ exactly once, the segment between $x$ and $y \cap S$ lying in $U . S$ is called a Cauchy hypersurface if $M$ is a Cauchy neighborhood over $S$.

Definition 2. A Cauchy problem ( 2 ; I, II, III, IV) consists in a 3-dimensional manifold $\Sigma$ and symmetric 2 -forms I, II, III, IV in $\Sigma$, I being definite, III and IV being tracefree, obeying the constraint equations $B_{n n}=0, B_{n i}=0$ in the appropriate interpretation.

Definition 3. A solution $(M, g, \lambda)$ of a Cauchy problem ( $\Sigma$; I, II, III, IV) consists in a space-time $(M, g)$ obeying Bach's field equations $B_{\alpha \beta}=0$ and an embedding $\lambda: \Sigma \rightarrow M$ such that $S:=\lambda(\Sigma)$ is a Cauchy hypersurface and that the data on $S$ induced from (I, II, III, IV) by $\lambda$ equal the data calculated from $g$. 
THEOREM 2 (Unicity result). For two solutions $\left(M^{\prime}, g^{\prime}, \lambda^{\prime}\right),\left(M^{\prime \prime}, g^{\prime \prime}, \lambda^{\prime \prime}\right)$ of a Cauchy problem ( $\Sigma$; I, II, III, IV) with $M^{\prime}, M^{\prime \prime}, \lambda^{\prime}, \lambda^{\prime \prime}, \Sigma$ being of class $C^{m+1}$ and $g^{\prime}, g^{\prime \prime} \in W^{m}(m \geqslant 6)$ there exists a Cauchy neighborhood $U^{\prime}$ over $S^{\prime}:=\lambda^{\prime}(\Sigma)$ and a Cauchy neighborhood $U^{\prime \prime}$ over $S^{\prime \prime}:=\lambda^{\prime \prime}(\Sigma)$ and a conformal mapping from $\left(U^{\prime}, g^{\prime}\right)$ to $\left(U^{\prime \prime}, g^{\prime \prime}\right)$.

Sketch of the proof. 1. Since $S^{\prime}, S^{\prime \prime}$ are Cauchy hypersurfaces in $M^{\prime}, M^{\prime \prime}$, there exist a $0<T \leqslant \infty$ and diffeomorphic embeddings $(-T, T) \times S^{\prime} \rightarrow M^{\prime},(-T, T) \times$ $\times S^{\prime \prime} \rightarrow M^{\prime \prime}$. Combining them with $\lambda^{\prime}, \lambda^{\prime \prime}$ we get embeddings $\Lambda^{\prime}, A^{\prime \prime}$ of $M$ := $(-T, T) \times \Sigma$ in $M^{\prime}, M^{\prime \prime}$. We diminish $M^{\prime}, M^{\prime \prime}$ to $\operatorname{im} \Lambda^{\prime}, \operatorname{im} \Lambda^{\prime \prime}$ and "ignore"; then $\lambda^{\prime}, A^{\prime \prime}$, thus identifying $M^{\prime}, M^{\prime \prime}$ with $M, S^{\prime}, S^{\prime \prime}$ with $S:=\{0\} \times \Sigma$ and $\lambda^{\prime}, \lambda^{\prime \prime}$ with the canonical embedding $\lambda: \Sigma \rightarrow S \subset M$.

2. There exists a conformal mapping $(\varphi, \Phi):\left(M, g^{\prime}\right) \rightarrow(M, \bar{g})$ preserving the data $\left(\lambda^{-1}\right)^{*}$ (I, II, III, IV) such that $\bar{g}:=\left(\varphi^{-1}\right)^{*}\left(\Phi_{g}\right)$ fulfills $\left.j^{3} \bar{g}\right|_{s}=\left.j^{3} g^{\prime \prime}\right|_{s}$. By "ignoring" $(\varphi, \Phi)$ we identify $g^{\prime}$ with $\bar{g}$. (Note that we write a conformal mapping as being composed by an isometry $\varphi$ and a conformal factor $\Phi$.)

3. Consider the linear Cauchy problem

$$
\square u+\frac{1}{6} R u=0,\left.\quad u\right|_{s}=1,\left.\quad \nabla u\right|_{s}=0
$$

with respect to $g^{\prime}, g^{\prime \prime}$. According to a theorem stated in [5] there exist Cauchy neighborhoods of $S$ and in these positive solutions $u^{\prime}, u^{\prime \prime} \in W^{m}$. The metrics $\bar{g}^{\prime}:=u^{\prime 2} g^{\prime}$, $\overline{\mathrm{g}}^{\prime \prime}:=u^{\prime \prime 2} \boldsymbol{g}^{\prime \prime}$ have vanishing scalar curvature $\bar{R}^{\prime}=0, \bar{R}^{\prime \prime}=0$. We write again $\boldsymbol{g}^{\prime}, \boldsymbol{g}^{\prime \prime}$ for $\bar{g}^{\prime}, \bar{g}^{\prime \prime}$.

4. Consider the local linear Cauchy problem

$$
\square u^{\mu}=0,\left.\quad u^{\mu}\right|_{s}=\left.x^{\mu}\right|_{s},\left.\quad \partial_{\alpha} u^{\mu}\right|_{s}=\delta_{\alpha}^{\mu}
$$

for determining four scalar fields $u^{\mu}(\mu=0,1,2,3)$ with respect to $g^{\prime}, g^{\prime \prime}$. Again according to [5] there exist coordinate (Cauchy) neighborhoods of an arbitrary point $y \in S$ and in these solutions $u^{\prime \mu}, u^{\prime \prime \mu} \in W^{m}$ representing harmonic coordinates. By mapping points with equal values of the harmonic coordinates we construct a local diffeomorphism $\psi_{y} \in W^{m+1}$ with $\left.j^{1} \psi_{y}\right|_{s}=\left.j^{1} \mathrm{id}\right|_{s}$. Then the $u^{\prime \prime \mu}(\mu=0,1,2,3)$ are harmonic coordinates with respect to both $\boldsymbol{g}^{\prime \prime}$ and $\tilde{\boldsymbol{g}}:=\left(\psi_{y}^{-1}\right)^{*} g^{\prime}$.

5. Bach's equations $B_{\alpha \beta}=0$ with respect to $\tilde{g}, g^{\prime \prime}$ in the coordinates $x^{\mu}:=u^{\prime \prime \mu}$ reduce to the strictly hyperbolic system $\breve{B}_{\alpha H}=0$. In (19) the summands (I, II, III, IV) and $(N, X)_{s}$ coincide with respect to $\tilde{g}, g^{\prime \prime}$ and $\left(j^{2} \Gamma^{\alpha}, j^{1} R\right)_{s}=(0,0)$ in our gauge. Hence we have $\left.j^{3} \tilde{g}_{\alpha \beta}\right|_{s}=\left.j^{3} g_{x \beta}^{\prime \prime}\right|_{s}$ and these data are of class $W^{m} \times W^{m-1} \times W^{m-2} \times$ $\times W^{m-3}$. According to a theorem stated in [8] there exists a coordinate (Cauchy) neighborhood of $y \in S$ where uniqueness $\tilde{\boldsymbol{g}}_{\alpha \beta}=\boldsymbol{g}_{\alpha \beta}^{\prime \prime}$ holds.

6. The local objects $\psi_{y}$ and $\tilde{g}_{\alpha \beta}=\boldsymbol{g}_{\alpha \beta}^{\prime \prime}$ can be pasted together to spatially global objects $\psi$ and $\tilde{g}=g^{\prime \prime}$. Now we do not longer "ignore" $A^{\prime}, A^{\prime \prime},(\varphi, \Phi), u^{\prime}, u^{\prime \prime}$ : These elements together with $\psi$ combine to the conformal mapping announced in Theorem 2.

Theorem 3 (Existence result). For a Cauchy problem $(\Sigma$; I, II, III, IV) with $\Sigma$ of class $C^{m+1}$ and (I, II, III, IV) $\in W^{m} \times W^{m-1} \times W^{m-2} \times W^{m-3}(m \geqslant 6)$ there exists a solution $(M, g, \lambda)$ with $M, \lambda$ of class $C^{m+1}$ and $g \in W^{m}$. 
Sketch of the proof. 1. We begin to work in the product manifold $R \times \Sigma$ of class $C^{m+1}$ and set

$$
\left(N, X, j^{2} I^{a}, j^{1} R\right):=(1,0,0,0)
$$

on $S:=\{0\} \times \Sigma$. According to (19) this initial gauge together with the minimal data (I, II, III, IV) constitutes maximal data $\left.j^{3} g_{\alpha \rho}\right|_{s}$ in each $\Sigma$ or $S$-coordinate neighborhood.

2. According to the theorem stated in [2] there exist coordinate (Cauchy) neighborhoods of an arbitrary $y \in S$ and in each of these a solution $g_{\alpha \beta}=g_{\alpha \beta}\left(x^{\prime \prime}\right)$ $\in W^{m}$ of the strictly hyperbolic system $\check{B}_{\alpha \beta}=0$ having the initial values $\left.j^{3} g_{a \beta}\right|_{s}$ constructed under item 1.

3. Our local solutions $g_{\alpha \beta}$ can be shown to satisfy the gauges $\Gamma^{\alpha}=0, R=0$ :

(a) According to a theorem stated in [8] the linear Cauchy problem

$$
2 g^{\alpha \beta} \check{B}_{\alpha \beta} \equiv \square \check{R}=0,\left.\quad j^{1} \stackrel{\check{R}}{\left.\right|_{s}} \equiv j^{1}\left(R+\nabla_{\alpha} \Gamma^{\alpha}\right)\right|_{s}=0
$$

has the unique solution $\check{R}=0$.

(b) One can show (here $\alpha=n, i$ )

$$
\left.B_{n \alpha}\right|_{s}=0 \text { and }\left.\quad \check{B}_{n \alpha}\right|_{s}=\left.0 \Rightarrow \hat{B}_{n x}\right|_{s}=\left.0 \Rightarrow j^{3} \Gamma^{\alpha}\right|_{s}=0 \text {. }
$$

(c) Taking (a), (b) into account one can derive from $\nabla^{\beta} B_{\alpha \beta} \equiv 0$ and $\nabla^{\beta} \check{B}_{\alpha \beta}=0$ a linear Cauchy problem

$$
\square U+b\left(g^{\alpha \beta}, j^{1} R_{\alpha \beta \mu \nu}\right) j^{1} U=0,\left.\quad j^{1} U\right|_{s}=0
$$

for the set of function $U:=\left(I_{\alpha}^{\prime}, \hat{R}_{\alpha \beta}, \square \Gamma_{\alpha}-R_{\alpha}^{\beta} \Gamma_{\beta}\right)$. Again according to [8] we have the unique solution $U=0$. Thanks to the gauge $\Gamma_{\alpha}=0, R \equiv \breve{R}+\hat{R}=0$ our $g_{\alpha \beta}$ solves the full Bach equations $B_{\alpha \beta}=0$.

4. The initial data and the gauge $R=0$ freeze the conformal freedom. Following $P$. Günther [6] we freeze the freedom of isometries by prescribing the values of the unit tangent vectors of the geodesics orthogonal to $S$. Thus the local solutions become comparable and can be pasted together to a spatially global solution $g$ defined in some Cauchy neighborhood $M \subseteq R \times \Sigma$ over $S=\{0\} \times \Sigma$. Care is to be taken to the orders of regularity, because uniqueness theorems are to be applied on differential equation systems occurring in the procedure.

\section{References}

[1] R. B a c h, Zur Weylschen Relativitätstheorie und der Weylschen Erweiterung des Krümmungstensorbegriffs, Math. Z. 9 (1921), 110-135.

[2] Y. C h o q u e t-B r u h a t, Solutions $C^{\infty}$ d'equations hyperboliques non lineaires, C.R. Acad. Sci. Paris Sér. A 272 (1971), 386-388.

[3] B. Fi ed le r and R. S $\mathrm{ch}$ i $\mathrm{m} \mathrm{m}$ i $\mathrm{ng}$, Exact solutions of the Bach field equations of general relativity, Rep. Mathematical Phys. 17 (1980), 15-36.

[4] B. F i e d l e r, Global $(2,2)$-decomposable solutions of the Bach equations of general relativity, Studia Sci. Math. Hungar. (to appear).

[5] A. E. Fis che r and J. E. M a r s d e n, General relativity, partial differential equations, and dynamical systems, AMS Proc. Symp. Pure Math. 23 (1973), 309-327. 
[6] P. G ü n t h e r, Uber das Cauchysche Problem für die Bachschen Feldgleichungen, Math. Nachr. 69 (1975), 39-56.

[7] -, Sprungrelationen und zugehörige Transportgleichungen für die Bachschen Feldgleichungen, Z. Angew. Math. Mech. 58 (1978), 429-434.

[8] S. W. H a w k ing and G. F. R. E. Il is, The large-scale structure of space-time, Cambridge 1973.

[9] K. S. S t e ll e, Renormalization of higher-derivative quantum gravity, Phys. Rev. D16 (1977), 953-969.

Presented to the Semester

Differential Geometry

(September 17-December 15, 1979) 